\title{
Psicoterapia familiar en pacientes con hipertensión no controlada. Estudio preliminar
}

\author{
Family psychotherapy in patients with uncontrolled hypertension. Preliminary study \\ Lidia V. Puga-Garfias, Oliva Mejía-Rodríguez², Esther G. Kanán-Cedeño , Pedro Caballero-Díaz³, \\ Martha M. Medellín-Fontes ${ }^{3}$ y Alain R. Rodríguez-Orozco ${ }^{4 *}$ \\ ${ }^{1}$ Unidad de Medicina Familiar 80, Instituto Mexicano del Seguro Social; ${ }^{2}$ Delegación del Instituto Mexicano del Seguro Social, Morelia; ${ }^{3}$ Facultad \\ de Psicología, Universidad Michoacana de San Nicolás de Hidalgo; ${ }^{4}$ Facultad de Ciencias Médicas y Biológicas Dr. Ignacio Chávez, Universidad \\ Michoacana de San Nicolás de Hidalgo. Morelia, Michoacán, México
}

\begin{abstract}
Resumen
Antecedentes: La psicoterapia familiar ha demostrado tener un impacto positivo en la evolución de enfermos con trastornos psicosomáticos, $y$ en aquellos con hipertensión arterial arterial pudiera mejorar el nivel de control de la enfermedad. Objetivo: Evaluar el impacto de una intervención en psicoterapia familiar en pacientes con hipertensión arterial sistémica no controlada. Método: Estudio con grupos comparados. 15 pacientes hipertensos no controlados, asignados a dos grupos: un de intervención, con 10 pacientes que recibieron psicoterapia familiar, y otro de control con 5 pacientes. En ambos grupos se continuó con el tratamiento farmacológico indicado por expertos. Se compararon ambos grupos antes y después de la intervención respecto a calidad de vida (InCaViSa), funcionamiento familiar (Escala de Funcionamiento Familiar), presión arterial sistólica y diastólica, índice de masa corporal, glucosa, colesterol, triglicéridos, ácido úrico y creatinina. Resultados: Hubo una mejoría cualitativa en los dominios de la escala InCaViSa para estimar la calidad de vida en el grupo de intervención y no hubo cambios estadísticamente significativos entre los grupos en cuanto a funcionamiento familiar y peso corporal. Entre las variables bioquímicas, solo el colesterol mostró una reducción significativa $(p=0.47)$. Se encontró una mayor movilidad de la familia hacia comportamientos más funcionales en el grupo que recibió la intervención, y también en este grupo disminuyeron las cifras de presión sistólica y diastólica $(p<0.001)$, así como el uso de antihipertensivos, ansiolíticos y antidepresivos. Conclusiones: La intervención psicoterapéutica familiar puede favorecer el control de la presión arterial, reducir las dosis de antihipertensivos, ansiolíticos y antidepresivos, y ayudar en la transición a estados de mejor funcionalidad familiar.
\end{abstract}

Palabras clave: Hipertensión arterial sistémica. Psicoterapia familiar. Hipertensión no controlada.

\begin{abstract}
Background: Family psychotherapy has been shown to have a positive impact on the evolution of patients with psychosomatic disorders, and in those with arterial hypertension it could improve the level of control of the disease Objective: To evaluate the impact of an intervention in family psychotherapy in patients with uncontrolled systemic arterial hypertension. Method: Study with comparative groups. 15 uncontrolled hypertensive patients, assigned to 2 groups: an intervention group of 10 patients who received family psychotherapy and a control group of 5 patients. In both groups, the pharmacological
\end{abstract}

Correspondencia:

*Alain R. Rodríguez-Orozco

E-mail: alain.rodriguez@umich.mx

DOI: 10.24875/ACM.20000505
Disponible en internet: $29-10-2021$ Arch Cardiol Mex. 2021;91(4):396-406 www.archivoscardiologia.com 1405-9940 / ( 2021 Instituto Nacional de Cardiología Ignacio Chávez. Publicado por Permanyer. Este es un artículo open access bajo la licencia CC BY-NC-ND (http://creativecommons.org/licenses/by-nc-nd/4.0/). 
treatment indicated by experts was continued. Both groups were compared before and after the intervention regarding quality of life (InCaViSa), family functioning (Family Functioning Scale), systolic and diastolic blood pressure, body mass index and glucose, cholesterol, triglycerides, uric acid and creatinine. Results: There was qualitative improvement in the domains of the InCaViSa scale to estimate quality of life in the intervention group and there were no statistically significant changes between the groups in family functioning or in body weight. Among the biochemical variables, only cholesterol showed a significant reduction $(p=0.47)$. Greater mobility of the family towards more functional behaviors was found in the group that received the intervention, and also in this group, systolic and diastolic blood pressure values decreased $(p<0.001)$, and the use of antihypertensive, anxiolytic and antidepressant drugs, as well. Conclusions: Family psychotherapeutic intervention can favor the control of blood pressure, reduce the doses of antihypertensive, anxiolytic and antidepressant drugs and it can help to transition to better functionally family states.

Key words: Systemic arterial hypertension. Family psychotherapy. Uncontrolled hypertension.

\section{Introducción}

Es frecuente el descontrol en los pacientes con hipertensión arterial sistémica a pesar de los cambios continuos en su manejo farmacológico y dietético. En este grupo de pacientes resulta conveniente evaluar la aplicación de formas de intervención no farmacológicas, y las intervenciones familiares en salud mental son una buena alternativa disponible y a bajo costo.

Desde la orientación sistémica de la terapia familiar, cuando hay un miembro de la familia que padece una enfermedad crónica ocurre una readaptación del resto de los miembros, lo cual con frecuencia implica cambios en las interacciones y modificaciones en la dinámica y la estructura de la familia, que una vez detectadas pueden usarse para proponer intervenciones psicoterapéuticas.

El tratamiento integral de la hipertensión arterial requiere la adopción de acciones farmacológicas y dietéticas, y cambios en el estilo de vida que comprometen al entorno familiar y a la habilidad para manejar las emociones.

La terapia estructural de familia es un tratamiento de acción enfocado en el sistema familiar que permite la exploración de las interacciones de los miembros de la familia1. Según Minuchin, referido por Eguiluz, y una transformación de la estructura familiar permitirá alguna posibilidad de cambio².

El terapeuta realiza una intervención en la familia con el objetivo de promover un cambio en la organización de esta hasta provocar una alteración en el contexto que alimenta al síntoma. El trabajo del terapeuta familiar con frecuencia se enfoca en el fortalecimiento de los subsistemas familiares, interviniendo en el desmantelamiento de alianzas y coaliciones que anclan el síntoma a un entorno psicosomático condicionante de la enfermedad.
Este trabajo tiene como objetivo evaluar el impacto de una intervención psicoterapéutica familiar en el control de la presión arterial y en la mejora del funcionamiento familiar y la calidad de vida de los pacientes con hipertensión arterial no controlada.

\section{Método}

\section{Tipo de estudio}

Estudio de intervención con grupos comparados.

\section{Población en estudio}

Pacientes con hipertensión no controlada en una unidad de medicina familiar en México.

\section{Criterios de inclusión}

De tres consultorios de medicina familiar se tomaron los pacientes con más de 5 años de diagnóstico de hipertensión arterial, entre junio y diciembre del año 2010, de ambos sexos, entre 18 y 70 años de edad, y con presión arterial sistémica $\geq 140 / 90 \mathrm{mmHg}$ en al menos cuatro de siete mediciones diarias la semana anterior, indicativo de descontrol según la Guía Mexicana de Práctica Clínica, IMSS-076-08³ a pesar de su correcta adherencia al tratamiento en el último mes (evaluada por la prueba de Morisky Green, por respuestas No/Sí/No/No a las cuatro preguntas del cuestionario) ${ }^{4}$.

No se incluyeron pacientes con trastornos psiquiátricos ni que recibieran terapia psicológica. Se excluyeron los pacientes que vivían solos y aquellos que no pudieran ser acompañados por un familiar conviviente a las sesiones de psicoterapia familiar. Se eliminaron las familias que decidieron retirarse del estudio en cualquiera de sus etapas y aquellas que no cumplieron 
con el $80 \%$ de asistencia a las sesiones psicoterapéuticas, así como las familias de pacientes que no asistieron a citas para la realización de estudios de laboratorio iniciales. Finalmente se incluyeron 15 familias de pacientes con hipertensión no controlada, de las cuales 10 recibieron psicoterapia familiar que incluyó al paciente con hipertensión no controlada, el cual además mantuvo su tratamiento farmacológico indicado por el médico internista o el cardiólogo, y las otras 5 familias integraron el grupo control, donde el paciente con hipertensión no controlada se mantuvo con el tratamiento farmacológico indicado por el médico internista 0 el cardiólogo.

Previo a la intervención y 1 mes después de concluida, se realizaron las siguientes evaluaciones:

- Mediciones clínicas y antropométricas: presión arterial sistólica, presión arterial diastólica e índice de masa corporal.

- Mediciones bioquímicas: se tomó una muestra sanguínea citando al paciente a las 7:00 a.m. con previo ayuno de 12 horas, y se determinaron la glucosa, el colesterol, los triglicéridos, el ácido úrico y la creatinina.

\section{Instrumentos}

- Cuestionario de Evaluación del Funcionamiento Familiar (EFF), que evalúa la percepción que los miembros de la familia tienen acerca de su funcionamiento. Es un cuestionario tipo Likert con cinco opciones de respuesta: 1 = totalmente en desacuerdo, $2=$ en desacuerdo, $3=$ ni de acuerdo ni en desacuerdo, $4=$ de acuerdo y $5=$ totalmente de acuerdo. Está integrado por 40 reactivos distribuidos en seis dimensiones: resolución de problemas, comunicación, roles, involucramiento afectivo, respuestas afectivas y control de conducta. Para la evaluación, los reactivos se agrupan en seis factores, cada uno con un punto de corte que permite evaluar cuán funcional 0 disfuncional es cada factor. Los factores son: involucramiento afectivo funcional con 17 reactivos y punto de corte de 51, involucramiento afectivo disfuncional con 11 reactivos y punto de corte de 33 , patrones de comunicación disfuncionales con 4 reactivos y punto de corte de 12, patrones de comunicación funcionales con 3 reactivos y punto de corte de 9 , resolución de problemas con 3 reactivos y punto de corte de 9 , y patrones de control de conducta con 2 reactivos y punto de corte de 6 , y se acepta como positiva sobre ese puntaje ${ }^{5}$.
- Inventario de Calidad de Vida y Salud (InCaViSa), que estima la percepción de satisfacción del sujeto ante diversos aspectos de su vida cotidiana. Las áreas que evalúa este inventario son preocupaciones, desempeño físico, aislamiento, percepción corporal, funciones cognitivas, actitud ante el tratamiento, tiempo libre, vida cotidiana, familia y dependencia médica ${ }^{6}$.

Tanto el cuestionario InCaViSa como el EFF se sometieron a evaluación de la consistencia interna con alfa de Cronbach y la prueba de mitades, y se obtuvieron valores que permitían su aplicación en la muestra objeto de estudio.

\section{Intervención psicoterapéutica familiar}

Las sesiones de psicoterapia estuvieron a cargo de un equipo de psicólogos con formación en psicoterapia familiar y se realizaron en la cámara de Gesell de la Facultad de Psicología de la Universidad Michoacana de San Nicolás de Hidalgo.

Se programaron ocho sesiones de la siguiente forma: una sesión por semana, con una duración de 2 horas aproximadamente cada una, y las sesiones se filmaron con el consentimiento de la familia para analizarlas por los médicos y los psicoterapeutas.

A continuación, se describe lo realizado en casa sesión:

1. Evaluación inicial: se aplicaron al paciente el cuestionario EFF, para determinar la percepción de este sobre su familia, y el InCaViSa.

2. Entrevista familiar: se citó a la familia conviviente del paciente con el objetivo de recopilar información sobre la estructura y la dinámica familiar, se exploró el sistema familiar de manera interactiva, se integró la información para la formulación de hipótesis y se propició el desarrollo del trabajo en grupo con la prescripción de tareas.

3. Explorar los recursos de la familia: se exploraron las redes de apoyo internas y externas con las que contaba la familia, para promover su fortalecimiento $y$ propiciar la creación de nuevos recursos para el apoyo del paciente hipertenso.

4. Explorar la comunicación de emociones en la familia: se estudió la comunicación en la familia para determinar no solo el nivel de contenido con que se comunican, sino también el nivel de relación con que lo hacen.

5. Explorar roles familiares: se determinaron los papeles (roles) de los miembros de la familia y sus contribuciones a la integración familiar, y se trabajó en 
la reestructuración de los roles correspondientes a cada uno de los integrantes de la familia.

6. Trabajar el mito familiar: se investigó si la familia cuenta con mitos familiares y se determinó si estos influyen en la conducta de sus miembros.

7. Manejo de los recursos familiares y afrontamiento: se evaluó la utilización de los recursos internos y externos con que la familia cuenta y su permeabilidad para recibir influencias del medio y modificarlo, se fomentó el uso de actitudes positivas y equilibrio emocional, y la mejora de las relaciones sociales.

8. Evaluación final: se aplicaron de nuevo el cuestionario EFF y el inventario InCaViSa y se determinaron los cambios logrados durante la terapia.

\section{Análisis estadístico}

La fiabilidad de los instrumentos empleados se evaluó con alfa de Cronbach y fue confirmada por el método de Spearman-Brown. El impacto de la intervención en la presión arterial, la somatometría y las variables bioquímicas se analizó con la prueba t de Student para muestras pareadas. Se almacenaron los datos en una base creada en SPSS versión 18 para Windows.

\section{Resultados}

La tabla 1 muestra las características generales de la población, tanto del grupo de intervención como del control. Ambos fueron homogéneos y por tanto objeto de comparación con las determinaciones propuestas.

\section{Variables clínicas y antropométricas}

La presión arterial sistólica tuvo una tendencia hacia una disminución progresiva desde la segunda sesión del programa psicoterapéutico. Este grupo inició con $140.0 \pm 2.98 \mathrm{mmHg}$ antes de comenzar la psicoterapia $y$ terminó con $121.60 \pm 2.96 \mathrm{mmHg}(p<0.0001), 1$ mes después de concluido el programa psicoterapéutico. En este mismo grupo y en el mismo periodo de tiempo, la presión arterial diastólica varió de $91.0 \pm 1.79 \mathrm{mmHg}$ al inicio del tratamiento hasta $74.80 \pm 2.46 \mathrm{mmHg}$ $(p<0.0001)$. El grupo que no recibió psicoterapia tendió a elevar los valores de presión arterial, tanto sistólica como diastólica, aunque los cambios no fueron estadísticamente significativos al comparar las mediciones al inicio y 1 mes después de terminada la intervención en el grupo que recibió psicoterapia. No se encontraron diferencias estadísticamente significativas en el índice de masa corporal al inicio ni 1 mes
Tabla 1. Características generales de ambos grupos al momento del reclutamiento

\begin{tabular}{|l|c|c|c|}
\hline Variable & Control & Intervención & $\mathbf{p}$ \\
\hline Edad (años) & $60.60 \pm 4.37$ & $56.50 \pm 3.63$ & 0.514 \\
\hline $\begin{array}{l}\text { Presión arterial } \\
\text { sistólica (mmHg) }\end{array}$ & $140.0 \pm 4.47$ & $140.0 \pm 2.98$ & 0.574 \\
\hline $\begin{array}{l}\text { Presión arterial } \\
\text { diastólica (mmHg) }\end{array}$ & $94.0 \pm 0.4$ & $91.0 \pm 1.79$ & 0.135 \\
\hline $\begin{array}{l}\text { Tiempo de evolución } \\
\text { (años) }\end{array}$ & $14.10 \pm 3.52$ & $14.20 \pm 3.04$ & 0.393 \\
\hline
\end{tabular}

después de concluida la intervención psicoterapéutica en ninguno de los grupos de estudio: el grupo intervención pasó de $32.40 \pm 1.70$ a $33.04 \pm 2.27$, y el grupo control pasó de $30.72 \pm 3.07$ a $29.58 \pm 3.18(p=0.081)$.

\section{Variables bioquímicas}

En la tabla 2 se muestran las variables bioquímicas de ambos grupos. Solo se encontró diferencia estadísticamente significativa entre las cifras de colesterol antes y después de la intervención en el grupo que recibió psicoterapia $(p=0.047)$.

\section{Tratamiento farmacológico}

La tabla 3 ilustra el efecto del programa psicoterapéutico en la reducción de dosis de medicamentos en cada grupo de estudio. Llama la atención la reducción de dosis de antihipertensivos, ansiolíticos y antidepresivos en el grupo de intervención en comparación con el que no recibió psicoterapia.

\section{Funcionalidad familiar y calidad de vida en salud}

Se evaluó la fiabilidad del cuestionario EFF para estimar el funcionamiento familiar y se encontró un puntaje global con alfa de Cronbach de 0.625 , y de 0.657 con la prueba de mitades. La tabla 4 muestra los cambios obtenidos en cada grupo para cada dominio del instrumento para estimar el funcionamiento familiar. Aunque 1 mes después de concluida la intervención psicoterapéutica no se encontraron cambios estadísticamente significativos para ninguno de los dominios de la funcionalidad familiar en ambos grupos de estudio, sí se vio que varios pacientes de este grupo mejoraron cualitativamente su funcionalidad en algunos dominios 
Tabla 2. Variables bioquímicas

\begin{tabular}{|l|c|c|c|c|c|c|}
\hline \multirow{2}{*}{ Variable } & \multicolumn{2}{|c|}{ Grupo de intervención } & \multirow{2}{*}{$\mathbf{p}$} & \multicolumn{2}{|c|}{ Grupo control } & \multirow{2}{*}{$\mathbf{p}$} \\
\cline { 2 - 7 } & Basal & Final & & Basal & Final \\
\hline Glucosa $(\mathrm{mg} / \mathrm{dl})$ & $98.30 \pm 8.3$ & $101.70 \pm 12.5$ & 0.355 & $131.20 \pm 51.8$ & $128.61 \pm 29.6$ & 0.891 \\
\hline Urea $(\mathrm{mg} / \mathrm{dl})$ & $34.20 \pm 6.4$ & $32.75 \pm 8.6$ & 0.669 & $26.20 \pm 9.3$ & $40.40 \pm 38.9$ & 0.357 \\
\hline Creatinina $(\mathrm{mg} / \mathrm{dl})$ & $1.08 \pm 0.7$ & $0.75 \pm 0.1$ & 0.468 & $0.90 \pm 0.45$ & $0.78 \pm 0.1$ & 0.330 \\
\hline Ácido úrico $(\mathrm{mg} / \mathrm{dl})$ & $6.00 \pm 1.2$ & $5.67 \pm 0.7$ & 0.512 & $6.08 \pm 1.7$ & $6.62 \pm 2.4$ & 0.702 \\
\hline Colesterol $(\mathrm{mg} / \mathrm{dl})$ & $201.10 \pm 46.2$ & $178.40 \pm 38.7$ & 0.047 & $155.0 \pm 83.2$ & $167.60 \pm 48.4$ & 0.797 \\
\hline Triglicéridos $(\mathrm{mg} / \mathrm{dl})$ & $191.30 \pm 119.0$ & $187.80 \pm 67.3$ & 0.921 & $170.60 \pm 42.5$ & $131.0 \pm 35.0$ & 0.250 \\
\hline
\end{tabular}

del cuestionario EFF, a diferencia del grupo control, en el que la tendencia fue hacia el estancamiento de las relaciones entre los miembros de la familia.

En la tabla 5 se muestra el impacto de la intervención psicoterapéutica en la calidad de vida y la salud cuando se comparan los grupos de control y de intervención. Se evaluó la fiabilidad del cuestionario InCaViSa para estimar el funcionamiento familiar y se encontró un puntaje global con alfa de Cronbach de 0.825, y de 0.753 con la prueba de mitades.

El grupo que recibió la intervención tendió a una mejora cualitativa en cada uno de los dominios de InCaViSa, al pasar de estados menos funcionales a más funcionales. Llama la atención la disminución de las preocupaciones y del aislamiento, el mejor uso de las redes sociales y del tiempo libre, la mejora en la actitud ante el tratamiento y en la relación con el médico, y la mejora en las funciones cognitivas, lo cual puede relacionarse con la mejor respuesta terapéutica en este grupo.

\section{Discusión}

Todos los pacientes que se sometieron a la intervención psicoterapéutica mejoraron el control de la presión arterial, tanto sistólica como diastólica. Estos cambios en el grupo de intervención propiciaron una disminución de las dosis de antihipertensivos y que el tratamiento con ansiolíticos y antidepresivos fuera descontinuado en $3 / 5$ pacientes, y disminuido en los otros 2 que lo usaban, a diferencia del grupo control, en el que se incrementaron las dosis de antihipertensivos o se agregó alguno más para lograr el control de las cifras tensionales, o se continuaron o incrementaron las dosis de antidepresivos y ansiolíticos.

Los programas de intervención educativa en las familias han mostrado un impacto favorable en el control de la presión arterial hasta 3 años después de concluidos $^{4}$, y otros basados en psicoterapia cognitivo-conductual han mostrado un impacto favorable en la calidad de vida de los pacientes con hipertensión? Asimismo, se ha demostrado la utilidad de estimular el autocuidado y la autogestión para mejorar el control de la presión arterial en adultos ${ }^{8}$, y el apoyo social y la adaptabilidad familiar ${ }^{9}$.

En los pacientes con hipertensión no controlada debe investigarse la presencia de otros factores de riesgo cardiovascular, como dislipidemia, intolerancia a la glucosa e hiperuricemia ${ }^{10}$. La exploración de variables bioquímicas como el perfil de lípidos, la glucosa y la creatinina puede resultar útil para evaluar su contribución al descontrol y el pronóstico del enfermo. En este estudio, solo el colesterol disminuyó significativamente en el grupo que recibió la intervención, lo cual puede atribuirse a la mejoría en el autocuidado y la autogestión de la enfermedad por el paciente, y a mejoras en el apoyo familiar que puede favorecer la implementación de hábitos saludables de alimentación y ejercicio, aun cuando no se apreciaron cambios en el índice de masa corporal.

En el paciente adulto con hipertensión no controlada debe explorarse la dinámica familiar para caracterizar el ambiente psicosomático que alimenta la enfermedad. Desde este punto de vista, la percepción del paciente sobre el funcionamiento familiar constituye el primer acercamiento crítico del enfermo a la situación que en el núcleo familiar lo ancla a la enfermedad, pero también una ayuda a la temprana identificación de recursos que la familia puede proveer para ayudarle, lo cual se aborda en la psicoterapia familiar. Hace cerca de 30 años que Onnis ${ }^{11}$ describió a la familia psicosomática como aquella en la que es frecuente encontrar rigidez (resistencia al cambio), aglutinación (los 
Tabla 3. Tratamiento farmacológico

\begin{tabular}{|c|c|c|c|c|c|c|c|c|}
\hline \multicolumn{9}{|c|}{ Grupo de intervención } \\
\hline \multirow[t]{3}{*}{ Paciente } & \multicolumn{4}{|c|}{ Antihipertensivos } & \multicolumn{4}{|c|}{ Ansiolíticos y antidepresivos } \\
\hline & \multicolumn{2}{|c|}{ Iniciales } & \multicolumn{2}{|l|}{ Finales } & \multicolumn{2}{|c|}{ Iniciales } & \multirow{2}{*}{\multicolumn{2}{|c|}{$\frac{\text { Finales }}{\text { Medicamento }}$}} \\
\hline & Medicamento & Dosis & Medicamento & Dosis & Medicamento & Dosis & & \\
\hline 1 & $\begin{array}{l}\text { Enalapril } \\
\text { Clortalidona } \\
\text { Losartán }\end{array}$ & $\begin{array}{l}\mathrm{c} / 8 \mathrm{~h} \\
\mathrm{c} / 24 \mathrm{~h} \\
\mathrm{c} / 12 \mathrm{~h}\end{array}$ & $\begin{array}{l}\text { Losartán } \\
\text { Enalapril }\end{array}$ & $\begin{array}{l}\mathrm{c} / 24 \mathrm{~h} \\
\mathrm{c} / 12 \mathrm{~h}\end{array}$ & $\begin{array}{l}\text { Fluoxetina } \\
\text { Clonazepam }\end{array}$ & $\begin{array}{l}\mathrm{c} / 24 \mathrm{~h} \\
\mathrm{c} / 24 \mathrm{~h}\end{array}$ & \multicolumn{2}{|l|}{ Suspendido } \\
\hline 2 & $\begin{array}{l}\text { Losartán } \\
\text { Hidroclorotiazida }\end{array}$ & $\begin{array}{l}\mathrm{c} / 12 \mathrm{~h} \\
\mathrm{c} / 24 \mathrm{~h}\end{array}$ & $\begin{array}{l}\text { Losartán } \\
\text { Hidroclorotiazida }\end{array}$ & $\begin{array}{l}\mathrm{c} / 24 \mathrm{~h} \\
\mathrm{c} / 24 \mathrm{~h}\end{array}$ & Ninguno & -------- & \multicolumn{2}{|l|}{ Ninguno } \\
\hline 3 & $\begin{array}{l}\text { Telmisartán } \\
\text { Metoprolol } \\
\text { Hidroclorotiazida }\end{array}$ & $\begin{array}{l}\mathrm{c} / 12 \mathrm{~h} \\
\mathrm{c} / 8 \mathrm{~h} \\
\mathrm{c} / 24 \mathrm{~h}\end{array}$ & $\begin{array}{l}\text { Telmisartán } \\
\text { Metoprolol } \\
\text { Hidroclorotiazida }\end{array}$ & $\begin{array}{l}\mathrm{c} / 12 \mathrm{~h} \\
\mathrm{c} / 24 \\
\mathrm{~h} \\
\mathrm{c} / 24 \mathrm{~h}\end{array}$ & Clonazepam & $\mathrm{c} / 24 \mathrm{~h}$ & \multicolumn{2}{|l|}{ Ocasional } \\
\hline 4 & $\begin{array}{l}\text { Metoprolol } \\
\text { Clortalidona } \\
\text { Enalapril }\end{array}$ & $\begin{array}{l}\mathrm{c} / 12 \mathrm{~h} \\
\mathrm{c} / 24 \mathrm{~h} \\
\mathrm{c} / 8 \mathrm{~h}\end{array}$ & $\begin{array}{l}\text { Metoprolol } \\
\text { Clortalidona } \\
\text { Enalapril }\end{array}$ & $\begin{array}{l}\mathrm{c} / 12 \mathrm{~h} \\
\mathrm{c} / 24 \mathrm{~h} \\
\mathrm{c} / 24 \mathrm{~h}\end{array}$ & Ninguno & -------- & \multicolumn{2}{|l|}{ Ninguno } \\
\hline 5 & $\begin{array}{l}\text { Losartán } \\
\text { Captopril } \\
\text { Hidroclorotiazida }\end{array}$ & $\begin{array}{l}\mathrm{c} / 12 \mathrm{~h} \\
\mathrm{c} / 8 \mathrm{~h} \\
\mathrm{c} / 24 \mathrm{~h}\end{array}$ & $\begin{array}{l}\text { Losartán } \\
\text { Captopril } \\
\text { Hidroclorotiazida }\end{array}$ & $\begin{array}{l}\mathrm{c} / 12 \mathrm{~h} \\
\mathrm{c} / 24 \mathrm{~h} \\
\mathrm{c} / 24 \mathrm{~h}\end{array}$ & Fluoxetina & $\mathrm{c} / 24 \mathrm{~h}$ & \multicolumn{2}{|l|}{ Suspendido } \\
\hline 6 & $\begin{array}{l}\text { Captopril } \\
\text { Hidroclorotiazida } \\
\text { Propanolol }\end{array}$ & $\begin{array}{l}\mathrm{c} / 8 \mathrm{~h} \\
\mathrm{c} / 24 \mathrm{~h} \\
\mathrm{c} / 12 \mathrm{~h}\end{array}$ & $\begin{array}{l}\text { Captopril } \\
\text { Hidroclorotiazida } \\
\text { Propanolol }\end{array}$ & $\begin{array}{l}\mathrm{c} / 12 \mathrm{~h} \\
\mathrm{c} / 24 \mathrm{~h} \\
\mathrm{c} / 12 \mathrm{~h}\end{array}$ & Ninguno & -------- & \multicolumn{2}{|l|}{ Ninguno } \\
\hline 7 & $\begin{array}{l}\text { Atenolol } \\
\text { Clortalidona } \\
\text { Irbesartán }\end{array}$ & $\begin{array}{l}\mathrm{c} / 24 \mathrm{~h} \\
\mathrm{c} / 24 \mathrm{~h} \\
\mathrm{c} / 12 \mathrm{~h}\end{array}$ & $\begin{array}{l}\text { Atenolol } \\
\text { Clortalidona } \\
\text { Irbesartán }\end{array}$ & $\begin{array}{l}\mathrm{c} / 24 \mathrm{~h} \\
\mathrm{c} / 24 \mathrm{~h} \\
\mathrm{c} / 24 \mathrm{~h}\end{array}$ & Imipramina & $\mathrm{c} / 24 \mathrm{~h}$ & \multicolumn{2}{|l|}{ Suspendido } \\
\hline 8 & $\begin{array}{l}\text { Losartán } \\
\text { Captopril } \\
\text { Hidroclorotiazida }\end{array}$ & $\begin{array}{l}\mathrm{c} / 12 \mathrm{~h} \\
\mathrm{c} / 8 \mathrm{~h} \\
\mathrm{c} / 24 \mathrm{~h}\end{array}$ & $\begin{array}{l}\text { Losartán } \\
\text { Captopril } \\
\text { Hidroclorotiazida }\end{array}$ & $\begin{array}{l}\mathrm{c} / 24 \mathrm{~h} \\
\mathrm{c} / 24 \mathrm{~h} \\
\mathrm{c} / 24 \mathrm{~h}\end{array}$ & Ninguno & -------- & \multicolumn{2}{|l|}{ Ninguno } \\
\hline 9 & $\begin{array}{l}\text { Metoprolol } \\
\text { Clortalidona } \\
\text { Captopril }\end{array}$ & $\begin{array}{l}\mathrm{c} / 12 \mathrm{~h} \\
\mathrm{c} / 24 \mathrm{~h} \\
\mathrm{c} / 8 \mathrm{~h}\end{array}$ & $\begin{array}{l}\text { Metoprolol } \\
\text { Clortalidona } \\
\text { Captopril }\end{array}$ & $\begin{array}{l}\mathrm{c} / 12 \mathrm{~h} \\
\mathrm{c} / 24 \mathrm{~h} \\
\mathrm{c} / 24 \mathrm{~h}\end{array}$ & $\begin{array}{l}\text { Imipramina } \\
\text { Clonazepam }\end{array}$ & $\begin{array}{l}\mathrm{c} / 12 \mathrm{~h} \\
\mathrm{c} / 24 \mathrm{~h}\end{array}$ & $\begin{array}{l}\text { Imipramina } \\
\text { Clonazepam }\end{array}$ & $\begin{array}{l}\text { Suspendido } \\
\mathrm{c} / 24 \mathrm{~h}\end{array}$ \\
\hline 10 & $\begin{array}{l}\text { Metoprolol } \\
\text { Hidroclorotiazida }\end{array}$ & $\begin{array}{l}\mathrm{c} / 8 \mathrm{~h} \\
\mathrm{c} / 24 \mathrm{~h}\end{array}$ & $\begin{array}{l}\text { Metoprolol } \\
\text { Hidroclorotiazida }\end{array}$ & $\begin{array}{l}\mathrm{c} / 8 \mathrm{~h} \\
\mathrm{c} / 24 \mathrm{~h}\end{array}$ & Ninguno & ------ & \multicolumn{2}{|l|}{ Ninguno } \\
\hline \multicolumn{9}{|c|}{ Grupo control } \\
\hline \multirow[t]{3}{*}{ Paciente } & \multicolumn{4}{|c|}{ Antihipertensivos } & \multicolumn{4}{|c|}{ Ansiolíticos y antidepresivos } \\
\hline & \multicolumn{2}{|c|}{ Iniciales } & \multicolumn{2}{|l|}{ Finales } & \multicolumn{2}{|c|}{ Iniciales } & \multicolumn{2}{|c|}{ Finales } \\
\hline & Medicamento & Dosis & Medicamento & Dosis & Medicamento & Dosis & \multicolumn{2}{|c|}{ Medicamento } \\
\hline 1 & $\begin{array}{l}\text { Enalapril } \\
\text { Hidroclorotiazida }\end{array}$ & $\begin{array}{l}\mathrm{c} / 8 \mathrm{~h} \\
\mathrm{c} / 24 \mathrm{~h}\end{array}$ & $\begin{array}{l}\text { Enalapril } \\
\text { Hidroclorotiazida } \\
\text { Losartán }\end{array}$ & $\begin{array}{l}\mathrm{c} / 8 \mathrm{~h} \\
\mathrm{c} / 24 \mathrm{~h} \\
\mathrm{c} / 12 \mathrm{~h}\end{array}$ & Clonazepam & $\mathrm{c} / 24 \mathrm{~h}$ & Clonazepam & $\mathrm{c} / 24 \mathrm{~h}$ \\
\hline 2 & $\begin{array}{l}\text { Telmisartán } \\
\text { Hidroclorotiazida } \\
\text { Enalapril }\end{array}$ & $\begin{array}{l}\mathrm{c} / 12 \mathrm{~h} \\
\mathrm{c} / 24 \mathrm{~h} \\
\mathrm{c} / 24 \mathrm{~h}\end{array}$ & $\begin{array}{l}\text { Telmisartán } \\
\text { Hidroclorotiazida } \\
\text { Felodipino }\end{array}$ & $\begin{array}{l}\mathrm{c} / 12 \mathrm{~h} \\
\mathrm{c} / 24 \mathrm{~h} \\
\mathrm{c} / 12 \mathrm{~h}\end{array}$ & Ninguno & -------- & $\begin{array}{l}\text { Fluoxetina } \\
\text { Clonazepam }\end{array}$ & $\begin{array}{l}\mathrm{c} / 24 \mathrm{~h} \\
\mathrm{c} / 24 \mathrm{~h}\end{array}$ \\
\hline 3 & $\begin{array}{l}\text { Captopril } \\
\text { Metoprolol } \\
\text { Hidroclorotiazida }\end{array}$ & $\begin{array}{l}\mathrm{c} / 8 \mathrm{~h} \\
\mathrm{c} / 12 \mathrm{~h} \\
\mathrm{c} / 24 \mathrm{~h}\end{array}$ & $\begin{array}{l}\text { Enalapril } \\
\text { Metoprolol } \\
\text { Hidroclorotiazida }\end{array}$ & $\begin{array}{l}\mathrm{c} / 8 \mathrm{~h} \\
\mathrm{c} / 12 \mathrm{~h} \\
\mathrm{c} / 24 \mathrm{~h}\end{array}$ & $\begin{array}{l}\text { Clonazepam } \\
\text { Fluoxetina }\end{array}$ & $\begin{array}{l}\mathrm{c} / 24 \mathrm{~h} \\
\mathrm{c} / 24 \mathrm{~h}\end{array}$ & $\begin{array}{l}\text { Clonazepam } \\
\text { Fluoxetina } \\
\text { Alprazolam }\end{array}$ & $\begin{array}{l}\text { Media c/24 h } \\
\mathrm{c} / 24 \mathrm{~h} \\
\text { Media c/24 h }\end{array}$ \\
\hline 4 & $\begin{array}{l}\text { Verapamilo } \\
\text { Enalapril } \\
\text { Hidroclorotiazida }\end{array}$ & $\begin{array}{l}\mathrm{c} / 12 \mathrm{~h} \\
\mathrm{c} / 12 \mathrm{~h} \\
\mathrm{c} / 24 \mathrm{~h}\end{array}$ & $\begin{array}{l}\text { Metoprolol } \\
\text { Enalapril } \\
\text { Verapamilo } \\
\text { Hidroclorotiazida }\end{array}$ & $\begin{array}{l}\mathrm{c} / 24 \mathrm{~h} \\
\mathrm{c} / 8 \mathrm{~h} \\
\mathrm{c} / 12 \mathrm{~h} \\
\mathrm{c} / 24 \mathrm{~h}\end{array}$ & Diazepam & $\mathrm{c} / 24 \mathrm{~h}$ & Diazepam & $\mathrm{c} / 24 \mathrm{~h}$ \\
\hline 5 & $\begin{array}{l}\text { Enalapril } \\
\text { Hidroclorotiazida }\end{array}$ & $\begin{array}{l}\mathrm{c} / 8 \mathrm{~h} \\
\mathrm{c} / 24 \mathrm{~h}\end{array}$ & $\begin{array}{l}\text { Losartán } \\
\text { Enalapril } \\
\text { Hidroclorotiazida }\end{array}$ & $\begin{array}{l}\mathrm{c} / 12 \mathrm{~h} \\
\mathrm{c} / 8 \mathrm{~h} \\
\mathrm{c} / 24 \mathrm{~h}\end{array}$ & Ninguno & --------- & Sertralina & $\mathrm{c} / 24 \mathrm{~h}$ \\
\hline
\end{tabular}

Las presentaciones fueron: hidroclorotiazida $25 \mathrm{mg}$, enalapril $10 \mathrm{mg}$, losartán $50 \mathrm{mg}$, telmisartán $40 \mathrm{mg}$, metoprolol $100 \mathrm{mg}$, clortalidona $50 \mathrm{mg}$, captopril $25 \mathrm{mg}$, propanolol $40 \mathrm{mg}$, atenolol $100 \mathrm{mg}$, irbesartán $150 \mathrm{mg}$, verapamilo $80 \mathrm{mg}$, fluoxetina $20 \mathrm{mg}$, clonazepam $2 \mathrm{mg}$, imipramina $25 \mathrm{mg}$, diazepam $10 \mathrm{mg}$, alprazolam $2 \mathrm{mg}$ y sertralina $50 \mathrm{mg}$. 
Tabla 4. Funcionalidad familiar

\begin{tabular}{|c|c|c|c|c|c|c|}
\hline \multirow[t]{2}{*}{ Dominio } & \multicolumn{3}{|c|}{$\begin{array}{l}\text { Grupo de intervención } \\
\qquad(\mathrm{n}=10)\end{array}$} & \multicolumn{3}{|c|}{$\begin{array}{l}\text { Grupo control } \\
(\mathrm{n}=5)\end{array}$} \\
\hline & Inicial & Final & $\mathbf{p}$ & Inicial & Final & $\mathbf{p}$ \\
\hline $\begin{array}{l}\text { Involucramiento afectivo funcional } \\
\text { Funcional } \\
\text { Med. funcional } \\
\text { Disfuncional }\end{array}$ & $\begin{array}{l}4 \\
5 \\
1\end{array}$ & $\begin{array}{l}7 \\
2 \\
1\end{array}$ & 0.638 & $\begin{array}{l}2 \\
3 \\
0\end{array}$ & $\begin{array}{l}1 \\
4 \\
0\end{array}$ & 0.490 \\
\hline $\begin{array}{l}\text { Involucramiento afectivo disfuncional } \\
\text { Funcional } \\
\text { Med. disfuncional } \\
\text { Disfuncional }\end{array}$ & $\begin{array}{l}1 \\
8 \\
1\end{array}$ & $\begin{array}{l}1 \\
8 \\
1\end{array}$ & 0.513 & $\begin{array}{l}1 \\
4 \\
0\end{array}$ & $\begin{array}{l}0 \\
5 \\
0\end{array}$ & 0.292 \\
\hline $\begin{array}{l}\text { Patrón de comunicación disfuncional } \\
\text { Funcional } \\
\text { Med. disfuncional } \\
\text { Disfuncional }\end{array}$ & $\begin{array}{l}1 \\
8 \\
1\end{array}$ & $\begin{array}{l}1 \\
9 \\
0\end{array}$ & 0.329 & $\begin{array}{l}0 \\
5 \\
0\end{array}$ & $\begin{array}{l}0 \\
5 \\
0\end{array}$ & 1.00 \\
\hline $\begin{array}{l}\text { Patrón de comunicación funcional } \\
\text { Funcional } \\
\text { Med. funcional } \\
\text { Disfuncional }\end{array}$ & $\begin{array}{l}1 \\
7 \\
2\end{array}$ & $\begin{array}{l}3 \\
7 \\
0\end{array}$ & 0.223 & $\begin{array}{l}3 \\
2 \\
0\end{array}$ & $\begin{array}{l}3 \\
2 \\
0\end{array}$ & 1.00 \\
\hline $\begin{array}{l}\text { Resolución de problemas } \\
\text { Funcional } \\
\text { Med. funcional } \\
\text { Disfuncional }\end{array}$ & $\begin{array}{l}1 \\
7 \\
2\end{array}$ & $\begin{array}{l}2 \\
7 \\
1\end{array}$ & 0.717 & $\begin{array}{l}1 \\
4 \\
0\end{array}$ & $\begin{array}{l}1 \\
4 \\
0\end{array}$ & 1.00 \\
\hline $\begin{array}{l}\text { Patrón de control de conducta } \\
\text { Funcional } \\
\text { Med. funcional } \\
\text { Disfuncional }\end{array}$ & $\begin{array}{l}4 \\
4 \\
2\end{array}$ & $\begin{array}{l}3 \\
5 \\
2\end{array}$ & 0.403 & $\begin{array}{l}2 \\
3 \\
0\end{array}$ & $\begin{array}{l}2 \\
3 \\
0\end{array}$ & 1.00 \\
\hline
\end{tabular}

Med.: medianamente.

miembros de la familia se sobreinvolucran recíprocamente), hiperprotección recíproca, en particular hacia el miembro enfermo, y evitación del conflicto, en parte porque tienen un bajo nivel de tolerancia a este. Tales características son frecuentes en las familias con enfermos con hipertensión no controlada y a su vez son susceptibles de modificarse con la psicoterapia familiar.

El análisis del paciente con hipertensión arterial no controlada no puede verse alejado de la compleja madeja de interacciones que establece con la familia. Tradicionalmente se ha considerado la hipertensión arterial como un modelo de enfermedad psicosomática, y las mejoras en los niveles de funcionamiento familiar pueden asociarse a mejoras en la percepción de la calidad de vida y en el control de la enfermedad por el paciente, al ser la familia la principal red de apoyo a este 12-14. $^{12}$

Un estudio correlacional iraní con un número pequeño de pacientes $(n=39)$ mostró que las variables del funcionamiento familiar solución de problemas, relaciones entre miembros, roles, involucramiento emocional, acompañamiento emocional, control de conducta y funcionamiento total no eran buenos predictores para la hipertensión ${ }^{15}$. Este estudio usó el Family Functioning Questionnaire. El hecho de no encontrar una relación de causalidad entre funcionamiento familiar y desarrollo de hipertensión arterial en este estudio no excluye que exista relación entre estas variables, e incluso se conoce que un entorno disfuncional condiciona la perpetuidad de la enfermedad; por ejemplo, el pobre apoyo social y la pobre regulación de las emociones se han asociado a reactividad aumentada a las hormonas del estrés en hipertensos ${ }^{16}$, y ambos factores socioculturales se establecen a partir de las relaciones sociales y en particular de las familiares ${ }^{17}$.

Por otro lado, unos mejores niveles de funcionamiento familiar se han relacionado con una menor prevalencia de problemas de salud en las familias, y ello ha permitido considerar a la familia como diana de las intervenciones terapéuticas para favorecer el control de las enfermedades crónicas, incluyendo la 
Tabla 5. Calidad de vida y salud

\begin{tabular}{|c|c|c|c|c|}
\hline \multirow[t]{2}{*}{ Dominio } & \multicolumn{2}{|c|}{$\begin{array}{l}\text { Grupo de intervención } \\
\qquad(\mathrm{n}=10)\end{array}$} & \multicolumn{2}{|c|}{$\begin{array}{l}\text { Grupo control } \\
\qquad(n=5)\end{array}$} \\
\hline & Inicial & Final & Inicial & Final \\
\hline $\begin{array}{l}\text { Preocupaciones } \\
\text { Muy baja } \\
\text { Baja } \\
\text { Normal } \\
\text { Alta } \\
\text { Muy alta }\end{array}$ & $\begin{array}{l}3 \\
5 \\
2 \\
0 \\
0\end{array}$ & $\begin{array}{l}0 \\
6 \\
4 \\
0 \\
0\end{array}$ & $\begin{array}{l}0 \\
1 \\
2 \\
1 \\
1\end{array}$ & $\begin{array}{l}0 \\
0 \\
1 \\
3 \\
1\end{array}$ \\
\hline $\begin{array}{l}\text { Desempeño físico } \\
\text { Muy baja } \\
\text { Baja } \\
\text { Normal } \\
\text { Alta } \\
\text { Muy alta }\end{array}$ & $\begin{array}{l}2 \\
3 \\
3 \\
2 \\
0\end{array}$ & $\begin{array}{l}1 \\
4 \\
3 \\
1 \\
1\end{array}$ & $\begin{array}{l}0 \\
1 \\
3 \\
0 \\
1\end{array}$ & $\begin{array}{l}0 \\
2 \\
2 \\
0 \\
1\end{array}$ \\
\hline $\begin{array}{l}\text { Aislamiento } \\
\text { Muy baja } \\
\text { Baja } \\
\text { Normal } \\
\text { Alta } \\
\text { Muy alta }\end{array}$ & $\begin{array}{l}4 \\
3 \\
1 \\
0 \\
2\end{array}$ & $\begin{array}{l}0 \\
5 \\
4 \\
0 \\
1\end{array}$ & $\begin{array}{l}0 \\
0 \\
4 \\
0 \\
1\end{array}$ & $\begin{array}{l}0 \\
0 \\
4 \\
1 \\
0\end{array}$ \\
\hline $\begin{array}{l}\text { Percepción corporal } \\
\text { Muy baja } \\
\text { Baja } \\
\text { Normal } \\
\text { Alta } \\
\text { Muy alta }\end{array}$ & $\begin{array}{l}3 \\
0 \\
7 \\
0 \\
0\end{array}$ & $\begin{array}{l}1 \\
3 \\
5 \\
0 \\
1\end{array}$ & $\begin{array}{l}2 \\
0 \\
2 \\
0 \\
1\end{array}$ & $\begin{array}{l}1 \\
2 \\
1 \\
1 \\
0\end{array}$ \\
\hline $\begin{array}{l}\text { Funciones cognitivas } \\
\text { Muy baja } \\
\text { Baja } \\
\text { Normal } \\
\text { Alta } \\
\text { Muy alta }\end{array}$ & $\begin{array}{l}2 \\
4 \\
2 \\
1 \\
1\end{array}$ & $\begin{array}{l}1 \\
1 \\
6 \\
1 \\
1\end{array}$ & $\begin{array}{l}0 \\
0 \\
2 \\
2 \\
1\end{array}$ & $\begin{array}{l}0 \\
2 \\
2 \\
0 \\
1\end{array}$ \\
\hline $\begin{array}{l}\text { Actitud ante el tratamiento } \\
\text { Muy baja } \\
\text { Baja } \\
\text { Normal } \\
\text { Alta } \\
\text { Muy alta }\end{array}$ & $\begin{array}{l}3 \\
4 \\
2 \\
0 \\
1\end{array}$ & $\begin{array}{l}0 \\
1 \\
5 \\
3 \\
1\end{array}$ & $\begin{array}{l}0 \\
3 \\
1 \\
0 \\
1\end{array}$ & $\begin{array}{l}0 \\
3 \\
2 \\
0 \\
0\end{array}$ \\
\hline $\begin{array}{l}\text { Tiempo libre } \\
\text { Muy baja } \\
\text { Baja } \\
\text { Normal } \\
\text { Alta } \\
\text { Muy alta }\end{array}$ & $\begin{array}{l}4 \\
1 \\
3 \\
0 \\
2\end{array}$ & $\begin{array}{l}1 \\
2 \\
5 \\
1 \\
1\end{array}$ & $\begin{array}{l}1 \\
0 \\
3 \\
0 \\
1\end{array}$ & $\begin{array}{l}0 \\
2 \\
2 \\
1 \\
0\end{array}$ \\
\hline $\begin{array}{l}\text { Vida cotidiana } \\
\text { Muy baja } \\
\text { Baja } \\
\text { Normal } \\
\text { Alta } \\
\text { Muy alta }\end{array}$ & $\begin{array}{l}4 \\
1 \\
4 \\
0 \\
1\end{array}$ & $\begin{array}{l}1 \\
2 \\
6 \\
1 \\
0\end{array}$ & $\begin{array}{l}1 \\
1 \\
1 \\
1 \\
1\end{array}$ & $\begin{array}{l}0 \\
2 \\
1 \\
2 \\
0\end{array}$ \\
\hline $\begin{array}{l}\text { Familia } \\
\text { Muy baja } \\
\text { Baja } \\
\text { Normal } \\
\text { Alta } \\
\text { Muy alta }\end{array}$ & $\begin{array}{l}3 \\
2 \\
4 \\
1 \\
0\end{array}$ & $\begin{array}{l}1 \\
3 \\
3 \\
3 \\
0\end{array}$ & $\begin{array}{l}0 \\
4 \\
1 \\
0 \\
0\end{array}$ & $\begin{array}{l}0 \\
4 \\
1 \\
0 \\
0\end{array}$ \\
\hline
\end{tabular}


Tabla 5. Calidad de vida y salud (Continuación)

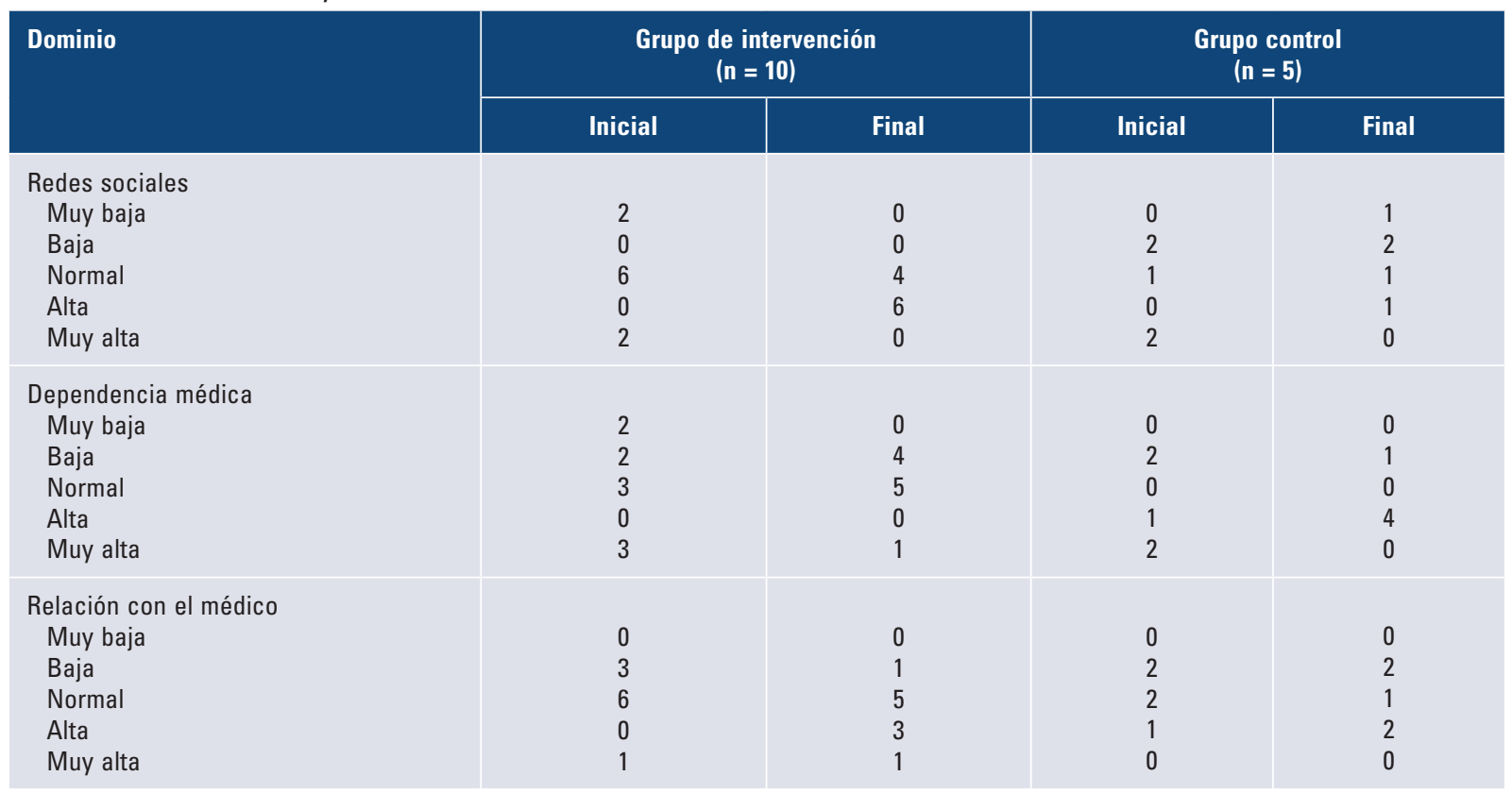

hipertensión arterial sistémica, todo esto en el contexto de la atención primaria de salud ${ }^{18}$.

En poblaciones de riesgo para padecer complicaciones por hipertensión de difícil control, como es el caso de las mujeres afroamericanas, se encontró una fuerte asociación entre funcionamiento familiar, en particular adaptabilidad familiar, y presión arterial sistólica ${ }^{9}$.

La relación entre el estado psicológico del paciente y el funcionamiento familiar con el mantenimiento de un clima emocional negativo que perpetúa el desarrollo de la enfermedad crónica ha sido muy comentada en la literatura. En el paciente, son factores susceptibles de modificar el ajuste emocional, la percepción de calidad de vida, la capacidad de afrontamiento y el análisis crítico de las creencias sobre la enfermedad. Durante el manejo psicoterapéutico es posible incidir en estos factores y en la gestión de recursos para enfrentar el problema en la familia. También resulta importante la evaluación de la función familiar frente a la enfermedad física a través de aspectos como la armonía, la calidad de la comunicación, las relaciones intergeneracionales y la capacidad de enfrentar la enfermedad, movilizando los recursos con los que la familia cuenta ${ }^{19}$.

Quizás uno de los estudios más conocidos de corte educativo, llevado a cabo hace más de 35 años, en familias para mejorar el control de la presión arterial, es uno en el que se estudió a 400 pacientes con una edad promedio de 54 años. Los miembros de la familia fueron asesorados y se les proporcionó un folleto con el propósito de involucrarlos en el manejo en el hogar de la presión arterial alta, lo cual incluyó ayuda al paciente con la adherencia al tratamiento y el cumplimiento de la medicación, la asistencia a citas médicas, la dieta y el control del peso. El grupo que recibió el programa educativo para promover el apoyo familiar presentó mejores resultados en el control de la presión diastólica hasta 3 años después4. Actualmente, las intervenciones psicoterapéuticas en familias y las de corte cognitivo-conductual han mostrado tener más impacto que aquellas en las que solo se implementan maniobras psicoeducativas, y más bien se incluyen estas en modelos de salud mental complejos, para tratar pacientes con hipertensión arterial sistémica no controlada.

En este estudio se evaluó la funcionalidad familiar antes y después de la psicoterapia familiar, y aunque no hubo diferencias estadísticamente significativas al comparar ambos grupos, sí se encontró una tendencia a mejorar el involucramiento afectivo funcional y el patrón de comunicación funcional en el grupo de intervención, lo que apunta al hecho de que con la psicoterapia familiar puede mejorarse la calidad de las interacciones con el integrante enfermo y fortalecer la comunicación para la resolución de problemas. El apoyo familiar y la adaptabilidad de la familia son aspectos importantes que deben considerarse en pacientes con hipertensión arterial no controlada ${ }^{9}$, así como la mejora en las 
relaciones entre los miembros de la familia. En cambio, en el grupo control se encontró un estancamiento en las funciones familiares evaluadas, lo que apunta a la conservación de un estado de rigidez que no permite evolucionar favorablemente el sistema familiar.

La calidad de vida de los pacientes tuvo cambios cualitativos positivos en todos los dominios de la prue$\mathrm{ba}$, al tender a mejorar la funcionalidad global en cada uno de ellos gracias a la intervención psicoterapéutica. Un estudio latinoamericano mostró una mejoría de la calidad de vida en todos los pacientes hipertensos sometidos a una intervención cognitivo-conductual ${ }^{7}$, y también se ha establecido que las intervenciones psicosociales en familias con un miembro con enfermedad crónica tienen efectos positivos tanto para la salud del paciente como para la de la familia ${ }^{20,21}$. Este tipo de intervenciones suelen tener un impacto benéfico en la salud mental del paciente y en la calidad de vida de este y de su familia. En particular son destacables los efectos positivos en el control de la ira, la depresión y la ansiedad, situaciones relacionadas con el pobre control de la hipertensión arterial, y también puede posibilitar la apertura a intervenciones alternativas, como cambios en la dieta y el uso de terapias complementarias $^{22}$. Todo esto puede estar involucrado en la disminución de las dosis de antihipertensivos, ansiolíticos y antidepresivos en el grupo intervención.

Otros aspectos que pueden influir en la mejoría del control de la presión arterial en el grupo de intervención son las mejoras en el autocuidado, incluyendo la alimentación, y la autogestión del paciente para entender mejor la enfermedad y llevar su tratamiento con el apoyo familiar, así como la contribución de las redes de apoyo y la posibilidad de favorecerse de los recursos instrumentales con los que cuenta la familia para enfrentar la enfermedad. En estudios posteriores, los autores proponen abordar la contribución de estos factores al control de la presión arterial en sujetos sometidos a psicoterapia familiar. Un estudio con 517 pacientes de edad avanzada con hipertensión arterial ha sugerido que mejorar el autocuidado y la autogestión tendría ser parte de un enfoque integral que debería tener en cuenta el apoyo social, la función familiar y el bienestar, y que es importante promover la calidad de vida en pacientes hipertensos de edad avanzada con bajo apoyo social y baja función familiar ${ }^{8}$.

\section{Conclusiones}

La psicoterapia de familia posibilita la apropiación de recursos psicológicos por el paciente y su familia para mejorar el control de la hipertensión arterial, ofrece a la familia una visualización de los conflictos internos y mejora la comunicación entre los miembros de la familia. Esto puede incidir en la mejora de la salud mental de la familia y en una percepción más crítica de la enfermedad por el paciente, y favorece su autogestión y autocuidado con el apoyo de la familia. Una intervención guiada por expertos en psicoterapia familiar puede disminuir las dosis de antihipertensivos, ansiolíticos y antidepresivos en los pacientes con hipertensión y mejorar el control de la enfermedad.

\section{Fortalezas del estudio}

Con una intervención en psicoterapia familiar realizada por expertos en una unidad de medicina familiar se logró mejorar el control de la presión arterial en sujetos no respondedores a tratamiento farmacológico, y se hizo con bajo costo. Se redujo el uso de antihipertensivos, ansiolíticos y antidepresivos. Existen muy pocos estudios similares con sujetos con hipertensión arterial sistémica realizados en la atención primaria, lo cual es más importante si se considera que en esta se atienden la mayoría de los pacientes con hipertensión arterial en las instituciones sanitarias mexicanas, que durante su formación el médico familiar ha recibido herramientas para explorar la relación familia-enfermedad y que está capacitado para trabajar con psicólogos en estudios de intervención similares que además ayudarían a disminuir los costos por atención médica en esta importante población de pacientes, que es grande, compleja y con altos niveles de descontrol. La intervención en psicoterapia familiar permite vislumbrar el marco relacional en el que aparece y se perpetúa la enfermedad en la familia, y proponer estrategias no farmacológicas para mejorar el nivel de control del paciente.

\section{Limitaciones del estudio}

Las limitaciones de este estudio son el pequeño número de familias incluidas y que no se explora el estado psicológico específico del individuo enfermo, sino que se enfoca en las relaciones de este con su familia y en su percepción de la calidad de vida para diseñar un programa psicoterapéutico familiar. Se trata de pocos casos, pero suficientes para documentar el impacto de la intervención sobre el control de la enfermedad. En próximos estudios consideraremos evaluar variables psicológicas que expliquen el resultado de la psicoterapia familiar en los pacientes con hipertensión arterial sistémica no controlada, y también identificar qué motivó 
en concreto el impacto sobre las variables bioquímicas, como la reducción del colesterol sérico. El seguimiento de esta cohorte, y la incorporación de nuevos casos, permitirá evaluar el efecto de la intervención en psicoterapia familiar en un periodo de tiempo más largo.

\section{Lo que aporta el estudio}

El estudio aporta al análisis del paciente con hipertensión arterial sistémica no controlada desde una perspectiva familiar para identificar variables susceptibles de modificación con psicoterapia familiar. Se puso énfasis en la percepción por el enfermo del funcionamiento familiar y de su calidad de vida y su salud como punto de partida para proponer el plan psicoterapéutico encaminado a mejorar el involucramiento de la familia en el tratamiento del paciente, y al fortalecimiento de la red de apoyo familiar para mejorar el control de la hipertensión arterial sistémica.

\section{Agradecimientos}

A Carlos Gómez Alonso, jubilado del Centro de Investigaciones Biomédicas de Michoacán, Instituto Mexicano del Seguro Social, Morelia, Michoacán, México, por su apoyo en el procesamiento estadístico de los datos.

\section{Financiamiento}

No se recibió financiamiento.

\section{Conflicto de intereses}

Ninguno que declarar.

\section{Responsabilidades éticas}

Aprobación por el Comité Local de Ética e Investigación en Salud. Registro ante el Comité Local de Investigación en Salud. R-2010-1602-45.

Protección de personas y animales. Los autores declaran que los procedimientos seguidos se conformaron a las normas éticas del comité de experimentación humana responsable y de acuerdo con la Asociación Médica Mundial, la Declaración de Helsinki y La Ley General de Salud de los Estados Unidos Mexicanos en materia de investigación en salud.

Confidencialidad de los datos. Los autores declaran que han seguido los protocolos de su centro de trabajo sobre la publicación de datos de pacientes.
Derecho a la privacidad y consentimiento informado. Los autores han obtenido el consentimiento informado de los pacientes y/o sujetos referidos en el artículo. Este documento obra en poder del autor de correspondencia.

\section{Bibliografía}

1. Umbarger-Carter C. Terapia familiar estructural. Buenos Aires: Amarrortu; 1999. p. 19-60.

2. Eguiluz LL. Terapia familiar, su uso hoy en día. México: Pax; 2004 p. $49-79$.

3. Guía Mexicana de Práctica Clínica, IMSS-076-08. Disponible en: http:// www.cenetec.salud.gob.mx/descargas/gpc/CatalogoMaestro/076-GCP HipertArterial1NA/GRR_HIPERTENSION.pdf

4. Morisky DE, DeMuth NM, Field-Fass M, Green LW, Levine DM. Evaluation of family health education to build social support for long-term control of high blood pressure. Health Education Quarterly. 1985;12:35-50.

5. Velasco M, Luna M. Cuestionario de Evaluación del Funcionamiento Familiar. En: Instrumentos de evaluación en terapia familiar y de pareja. México: Pax; 2006;46-68.

6. Riveros R, Sánchez J, Del Águila M. Inventario de Calidad de Vida y Salud (InCaViSa). México: Manual Moderno; 2009

7. Riveros A, Ceballos G, Laguna R, Sánchez-Sosa JJ. El manejo psicológico de la hipertensión esencial: efectos de una intervención cognitivo-conductual. Rev Latin Psicol. 2005;37:493-507.

8. Zhang X, Zheng Y, Qiu C, Zhao Y, Zang X. Well-being mediates the effects of social support and family function on self-management in elderly patients with hypertension. Psychol Health Med. 2020;25:559-71.

9. Brittain K, Taylor JY, Wu CY. Family adaptability and cohesion and high blood pressure among urban African American women. I Nurse Pract. 2010;6:786-93.

10. Mejía-Rodríguez O, Paniagua-Sierra R, Valencia-Ortiz MDR, Ruiz-García J, Figueroa-Núñez B, Roa-Sánchez V. Factores relacionados con el descontrol de la presión arterial. Salud Publica Mex. 2009;51:291-7.

11. Onnis L. Terapia familiar de los trastornos psicosomáticos. Barcelona: Paidós; 1990.

12. Rodríguez-Orozco AR, Kanán-Cedeño EG, Vázquez-Romero ME, Barbosa-Sánchez CE, Tejeda Cárdenas RG, León-Gutiérrez A. Asma y disfunción familiar. Un acercamiento a la medicina psicosomática. Alerg Asma Inmunol Pediatr. 2007:16:4-5.

13. Kanán-Cedeño GE, Rivera ME, Rodríguez-Orozco AR, López-Peñaloza J, Medellin-Fontes MM, Caballero-Díaz P. Funcionamiento familiar de los pacientes hipertensos con y sin la experiencia de la migración. Psicología y Salud. 2010;20:203-12.

14. Medellín-Fontes M, Rivera-Heredia ME, López-Peñaloza J, Kanán-Cedeño MG, Rodríguez-Orozco AR. Funcionamiento familiar y su relación con las redes de apoyo social en una muestra de Morelia, México. Salud Mental. 2012;35:147-54.

15. Manshaee G, Hariri M, Aqarashti Z, Khanbani F. Assessment of family functioning in patients with psychosomatic disorders (diabetes, hypertension, heart assessment). J Sociol Research. 2014;5:171-81.

16. Wirtz PH, von Känel R, Mohiyeddini C, Emini L, Ruedisueli K, Groessbauer S, et al. Low social support and poor emotional regulation are associated with increased stress hormone reactivity to mental stress in systemic hypertension. J Clin Endocrinol Metab. 2006;91:3857-65.

17. Rodríguez-Orozco AR, López-Peñaloza J, Kanán-Cedeño EG, Villalón-Santillán S, Caballero-Díaz P, Medellín-Fontes MM, et al. Familial system and allergic child. Notes about the role of the symptom in the familial functioning. Revista Alergia México. 2009;56:217-22.

18. García-Huidobro D, Puschel K, Soto G. Family functioning style and health: opportunities for health prevention in primary care. Br J Gen Pract. 2012;62:e198-203

19. Weihs K, Fisher L, Baird M. Families, health, and behavior: a section of the commissioned report by the Committee on Health and Behavior: Research, Practice, and Policy Division of Neuroscience and Behavioral Health and Division of Health Promotion and Disease Prevention Institute of Medicine, National Academy of Sciences. Fam Syst Health. 2002;20:7.

20. Hartmann M, Bäzner E, Wild B, Eisler I, Herzog W. Effects of interventions involving the family in the treatment of adult patients with chronic physical diseases: a meta-analysis. Psychother Psychosom. 2010;79: 136-48.

21. Campbell TL. The effectiveness of family interventions for physical disorders. J Marital Fam Ther. 2003;29:263-81.

22. Suquilvide-Miranda J, Rodríguez-Orozco AR, García-Pérez ME, Escudero-Hernandez L, Gómez-Alonso C, Cortés-Rojo C. Family functioning and the use of complementary therapies in patients with psoriasis. Atencion Primaria. 2019;52:435-6. 